\title{
Anti-apoptotic and pro-survival effect of exercise training on early aged hypertensive rat cerebral cortex
}

\author{
Yi-Jie Liu ${ }^{1,2,{ }^{*}}$, Zhen-Yang Cui ${ }^{3,}{ }^{*}$, Ai-Lun Yang ${ }^{4}$, Amadou W. Jallow ${ }^{5}$, Hai-Liang Huang ${ }^{6}$, \\ Chun-Lei Shan ${ }^{1,2}$, Shin-Da Lee ${ }^{1,3,5,7,8}$ \\ ${ }^{1}$ School of Rehabilitation Medicine, Shanghai University of Traditional Chinese Medicine, Shanghai, China \\ ${ }^{2}$ Institute of Rehabilitation Medicine, Shanghai University of Traditional Chinese Medicine, Shanghai, China \\ ${ }^{3}$ School of Rehabilitation Medicine, Weifang Medical University, Shandong, China \\ ${ }^{4}$ Institute of Sports Sciences, University of Taipei, Taipei, Taiwan \\ ${ }^{5}$ Department of Medical Laboratory and Biotechnology, Asia University, Taichung, Taiwan \\ ${ }^{6}$ College of Rehabilitation, Shandong University of Traditional Chinese Medicine, Shandong, China \\ ${ }^{7}$ Department of Physical Therapy, Graduate Institute of Rehabilitation Science, China Medical University, Taichung, \\ Taiwan \\ ${ }^{8}$ Department of Physical Therapy, Asia University, Taichung, Taiwan \\ *Equal contribution
}

Correspondence to: Shin-Da Lee, Chun-Lei Shan; email: shinda@mail.cmu.edu.tw, shanclhappy@163.com, https://orcid.org/0000-0002-2742-1281

Keywords: brain, early aged hypertension, neuroprotection, neuronal apoptosis

Received: October 29, $2020 \quad$ Accepted: July 23, 2021

Published: August 25, 2021

Copyright: (C) 2021 Liu et al. This is an open access article distributed under the terms of the Creative Commons Attribution License (CC BY 3.0), which permits unrestricted use, distribution, and reproduction in any medium, provided the original author and source are credited.

\section{ABSTRACT}

The anti-apoptotic and pro-survival effects of exercise training were evaluated on the early aged hypertensive rat cerebral cortex. The brain tissues were analysed from ten sedentary male Wistar Kyoto normotensive rats (WKY), ten sedentary spontaneously 12 month early aged hypertensive rats (SHR), and ten hypertensive rats undergoing treadmill exercise training (60 min/day, 5 days/week) for 12 weeks (SHR-EX). TUNEL-positive apoptotic cells, the expression levels of endonuclease $\mathbf{G}$ (EndoG) and apoptosis-inducing factor (AIF) (caspase-independent apoptotic pathway), Fas ligand, Fas death receptor, tumor necrosis factor (TNF)- $\alpha$, TNF receptor 1, Fas-associated death domain, active caspase-8 and active caspase-3 (Fas-mediated apoptotic pathways) as well as t-Bid, Bax, Bak, Bad, cytochrome c, active caspase 9 and active caspase-3 (mitochondria-mediated apoptotic pathways) were reduced in SHR-EX compared with SHR. Pro-survival Bcl2, Bcl-xL, p-Bad, 14-3-3, insulin-like growth factor (IGF)-1, pPI3K/PI3K, and PAKT/AKT were significantly increased in SHR-EX compared to those in SHR. Exercise training suppressed neural EndoG/AIF-related caspase-independent, Fas/FasL-mediated caspase-dependent, mitochondria-mediated caspase-dependent apoptotic pathways as well as enhanced $\mathrm{Bcl}-2$ family-related and IGF-1-related pro-survival pathways in the early aged hypertensive cerebral cortex. These findings indicated new therapeutic effects of exercise training on preventing early aged hypertension-induced neural apoptosis in cerebral cortex.

\section{INTRODUCTION}

Hypertension (HTN) is considered as the most prevalent and leading cause of most morbidities and mortalities in worldwide health [1]. Progressive brain damage is one of the adverse complications of hypertension [2]. A previous study revealed that hypertension-induced structural changes in the brain such as vascular remodeling, cerebral microbleeds, cerebral atrophy, and impaired cerebral autoregulation [3]. Some studies reported that chronic hypertension appeared to aggravate Parkinson's diseases, Alzheimer's diseases, dementia, and cognitive impairment $[4,5]$. Cerebral cortex apoptosis was found in a transgenic mouse model 
of Alzheimer's disease with cognitive dysfunction [6]. Neuroinflammation which was augmented in hypertension was also identified as a key determinant resulting in brain dysfunction [7]. Pro-inflammatory tumor necrosis factor (TNF) alpha also enhances neural cellular apoptotic pathway through binding tumor necrosis factor receptor 1 (TNFR1), a death receptor and harboring a death domain [8]. Moreover, high blood pressure deteriorates neuronal functions [9], and causes destruction on both small and large cerebral vessels leading to dementia and brain damage [10]. Therefore, strategies to control blood pressure would provide neurological benefits.

Exercise training had been known to lower the sympathetic activity and was beneficial for the prevention and treatment of hypertension and its cognitive impairments [11]. A study showed that aerobic exercise manifested both short-term and long-term effects on blood pressure control [12]. Exercise had demonstrated a neuroprotective effect in extensive studies and was recommended to counteract brain dysfunction in neurodegenerative disorders [13, 14]. Moreover, some studies revealed that chronic exercise training improved cognitive impairment as well as delayed the onset of neurodegenerative diseases including Alzheimer's disease and dementia $[15,16]$. There was various overwhelming literature confirming that exercise training improved the brain cortex and cerebellum AIF function as well as reduced oxidative stress and apoptotic related markers $[16,17]$. Decreased apoptotic neuronal cell death in the motor cortex was observed after exercise training which inhibited brain inflammation-induced motor impairment [18]. Pre-exercise training in traumatic brain areas significantly diminished lesion area, prevented neuronal loss, and reduced microglial activation in the cortex [19]. Numerous studies showed that voluntary running alone was adequate to enhance cell proliferation and survival, upgrade synaptic versatility and improve cognitive performance [20, 21]. However, the protective mechanism of exercise training on the cerebral cortex remains elusive.

Apoptosis is a form of programmed cell death and is regulated by diverse signalling pathways. However, the mechanism of neural apoptosis in cerebral cortex is complicated. Endonuclease G (EndoG) and apoptosisinducing factor (AIF) caspase-independence apoptotic pathway is one of the mechanisms responsible for initiating neural apoptosis [22]. The initiation of this pathway involves the release of Endo G and AIF from the mitochondria to translocate into the nucleus. The Endo $\mathrm{G}$ is responsible to trigger DNA fragmentation [23] whereas AIF causes nuclear changes and chromatin condensation [24]. Previous studies had shown that exercise training significantly reduced AIF expression and interrupted AIF migration to the nucleus in brain injury $[19,25]$. Currently, the effect of exercise training on Endo G/AIF neural cell death pathway remains unknown in the cerebral cortex of hypertension.

Extrinsic or Fas/FasL-initiated apoptotic pathway and intrinsic mitochondrial initiated apoptotic pathway were considered as the main mechanism in neural apoptosis [26]. Research has shown that Fas ligand binding to Fas receptor or TNF- $\alpha$ binding with TNFR1 receptors, death receptor Fas or tumor necrosis factor (TNF) receptors is directly involved in initiating Fas/FasL-mediated apoptotic pathway in neural apoptosis [27]. Initiation of the pathway involves activation of the death receptor Fas binding with Fas ligand and leads to the formation of death-inducing signalling complex (DISC). During the binding process of Fas receptor and Fas ligand, the adaptor protein Fas-associated death domain (FADD) and pro-caspase- 8 are recruited and induce Caspase- 8 activation. When caspase- 8 is activated, it will subsequently activate Caspase- 3 cleavage then induce apoptosis [26]. While the effect of exercise training on neural Fas-initiated apoptotic pathway in hypertensive cerebral cortex remains still unclear.

The mitochondria-mediated apoptotic pathway or intrinsic pathway in neural apoptosis involves the release of Cytochrome $c$. Interaction of either homodimers or heterodimers of $\mathrm{Bcl}-2$ family proteins composing of both pro- and anti-apoptotic proteins leads to the release of Cytochrome $c$ into the cytosol. Released Cytochrome $\mathrm{c}$ will interact with apoptosis protease activating factor-1 (Apaf-1) and pro-caspase-9 in the cytosol to form an apoptosome complex. By closed clustering and interacting with apoptosome, Cytochrome $\mathrm{c}$ leads to the activation of caspase- 9 from pro-caspase-9. Then, activated caspase- 9 will eventually cleave pro-caspase- 3 to produce the effector protease caspase-3 and initiate apoptosis [28]. The action of proapoptotic proteins is strongly controlled by members of the anti-apoptotic Bcl-2 family proteins such as Bcl-2 and $\mathrm{Bcl}-\mathrm{xL}$ and anti-apoptotic $\mathrm{Bcl}-2$ family proteins such as Bcl-2, Bcl-xL, p-Bad, and 14-3-3 [29]. However, the role of exercise training on neural mitochondriamediated apoptotic pathway in the hypertensive cerebral cortex is still unknown.

Insulin-like growth factor (IGF-1) plays an essential role in promoting cell proliferation, differentiation and inhibiting neural apoptosis through the activation of PI3K/AKT signaling pathway [30]. When IGF-1 activates phosphatidylinositol 3 kinase (PI3K), it will consequently activate the downstream of protein kinase B (AKT). The activation AKT promotes neural proliferation and regeneration [31, 32]. Phosphorylated AKT also interacts with Bcl-2 related pro-survival proteins to inhibit apoptosis [33]. 
The current study investigated whether exercise training could prevent neural apoptosis on early aged hypertensive cerebral cortex. Therefore, we hypothesized that exercise training might prevent hypertension-induced EndoG/AIF caspase-independent, Fas/FasL-mediated caspase-dependent and mitochondria-mediated caspasedependent apoptotic pathways also aggrandized related Bcl-2 family and IGF-1 pro-survival pathways in the cerebral cortex.

\section{RESULTS}

\section{Body weight and cortical characteristics}

There were no significant differences in body weight and brain weight among the three groups (WKY, SHR, and SHR-EX). The heart rate, systolic, diastolic and mean arterial blood pressure were higher in SHR group than WKY group. The heart rate, systolic blood pressure, diastolic blood pressure and mean arterial blood pressure were significantly decreased in SHREX compared with SHR group. The recorded pulse pressure was higher in SHR group compared with WKY and was decreased in SHR-EX compared with SHR group. To determine the effectiveness of exercise training among three groups, we measured the Citrate Synthase Activity. The Citrate Synthase Activity in the SHE-EX group was higher compared with SHR or WKY (Table 1).

\section{TUNEL-positive apoptotic cells of cerebral cortex}

To determine the degree of apoptosis in the cerebral cortex of WKY, SHR, and SHR-EX after exercise training, TUNEL assay and DAPI staining were measured. We observed that the number of TUNELpositive neural cells in the SHR group was greater than WKY, whereas, the number of TUNEL-positive neural cells in SHR-EX was lower than SHR group (Figure 1A, 1B).

\section{EndoG/AIF-related caspase-independent neural apoptotic pathway}

To determine the effect of exercise training on the neural EndoG/AIF-related caspase-independent apoptotic pathway in the cerebral cortex, the protein level of Endo $G$ and AIF in the three groups (WKY, SHR, and SHR-EX) were measured by western blot. The protein levels of EndoG and AIF in SHR were increased compared to WKY group. The protein levels of EndoG and AIF in SHR-EX group were decreased compared to SHR group, suggesting that exercise training could prevent neural apoptosis in the early aged hypertensive brain via EndoG and AIF pathways (Figure 2).
Upstream components of neural Fas/FasL-mediated caspase-dependent apoptotic pathway

To investigate whether exercise training can affect the neural Fas/FasL-mediated caspase-dependent apoptotic pathway, western blot was conducted to measure the protein level of Fas, FasL, and FADD from the excised cerebral cortex tissues of the WKY, SHR and SHR-EX group. The levels of TNF $\alpha$, TNF-R1, Fas, FasL, and FADD in SHR were increased compared to WKY group and those were decreased in SHR-EX compared with SHR group (Figure 3).

Upstream components of neural mitochondriamediated caspase-dependent apoptotic pathway

To examine the upstream components of the neural mitochondria-mediated caspase-dependent apoptotic pathway consisting of both pro-apoptotic and antiapoptotic of the Bcl-2 protein family, western blot analysis was conducted in the cortical tissues of the three groups including WKY, SHR, and SHR-EX. The levels of tBid, Bax, Bak, Bax/Bcl2 and Bax/Bcl-xL were higher in SHR group compared with WKY group as well as drastically lower in SHR-EX group than those in SHR group. The levels of Bcl2, Bcl-xL, $\mathrm{pBad}, \mathrm{pBad} / \mathrm{Bad}, 14-3-3$ in SHR group were significantly decreased compared with WKY group as well as those were significantly increased in SHR-EX group compared with SHR group, indicating that exercise training inhibited neural mitochondriamediated pro-apoptotic pathway and enhanced mitochondria-mediated anti-apoptotic pathway through these upstream components of mitochondria-mediated apoptotic pathway (Figure 4).

The downstream components of neural Fas/FasLmediated and mitochondria-mediated caspasedependent apoptotic pathway

The downstream components of Fas and mitochondriamediated caspase-dependent apoptotic pathway in the cerebral cortex in WKY, SHR, and SHR-EX were investigated by western blot. The protein levels of active Caspase-8 and active Caspase-3 (apoptotic pathway), cytosolic Cytochrome, Apaf-1, active Caspase-9, active Caspase3 (mitochondria-mediated caspase-dependent apoptotic pathway) in SHR group were increased compared with WKY group whereas those components were decreased in SHR-Ex group compared with SHR group, suggesting that exercise training could prevent neural apoptosis through these downstream components of Fas/FasL-mediated and mitochondria-mediated caspase-dependent apoptotic pathway (Figure 5). 
Table 1. Characteristics of WKY, SHR and SHR-EX groups.

\begin{tabular}{lccc}
\hline & WKY & SHR & SHR-EX \\
\hline Number of animals & 10 & 10 & 10 \\
Body weight, g & $398 \pm 9$ & $402 \pm 11$ & $396 \pm 13$ \\
Brain weight, g & $2.20 \pm 0.04$ & $2.25 \pm 0.06$ & $2.24 \pm 0.07$ \\
Heart rate, beats/min & $279 \pm 11$ & $390 \pm 17^{*}$ & $359 \pm 18^{* \#}$ \\
Systolic blood pressure, $\mathrm{mmHg}$ & $114 \pm 4$ & $193 \pm 5^{*}$ & $177 \pm 4^{* \#}$ \\
Diastolic blood pressure, $\mathrm{mmHg}$ & $87 \pm 7$ & $154 \pm 3^{*}$ & $136 \pm 4^{* \#}$ \\
Mean blood pressure, mmHg & $95 \pm 6$ & $167 \pm 3^{*}$ & $150 \pm 3^{* \#}$ \\
Pulse pressure, mmHg & $27 \pm 3$ & $39 \pm 2^{*}$ & $27 \pm 4^{* \#}$ \\
Citrate Synthase Activity & $1.58 \pm 0.04$ & $1.59 \pm 0.05$ & $1.93 \pm 0.07^{* \#}$ \\
$\left(\mu\right.$ mol min $\left.^{-1} \mathrm{~g} \mathrm{wet} \mathrm{wt}^{-1}\right)$ & & & \\
\hline
\end{tabular}

Values are means \pm SEM amount a normotensive Wistar Kyoto (WKY) group, a spontaneously early aged hypertensive (SHR) group and a hypertension with 12 weeks Exercise training (SHR-EX) group. ${ }^{*} p<0.05$ Significant differences between WKY and SHR or between WKY and SHR-EX group. ${ }^{\#} p<0.05$ significant differences between SHR group and SHR-EX group.

\section{The neural IGF-1-related pro-survival pathway}

To explore whether exercise training enhances the prosurvival components of neural apoptosis in the early aged hypertensive cortex, western blot was conducted to measure the protein level of IGF-1, PI3K, pPI3K, AKT and $\mathrm{pAKT}$ in the cerebral cortex. The expression levels of IGF-1, PI3K, pPI3K, AKT, and pAKT in SHR group were significantly decreased compared with WKY group. A significant increase of IGF-1, PI3K, pPI3K, AKT, and pAKT in SHR-EX group was observed compared with SHR group (Figure 6).

\section{DISCUSSION}

\section{Major findings}

The main new findings were outlined as follows: 1) Early aged hypertension-activated neural EndoG/AIFrelated caspase-independent, Fas/FasL-mediated caspase-dependent, and mitochondria-mediated caspase-dependent apoptotic pathways as well as suppressed Bcl-2 family-related and IGF-1-related prosurvival pathways in the cerebral cortex. 2) Exercise training decreased early aged hypertension-induced TUNEL positive apoptotic cells in the cerebral cortex. 3) Exercise training decreased early aged hypertension hypertension-induced neural EndoG/AIF-related caspase-independent apoptotic pathways, which was supported by the expression levels of EndoG and AIF in the cerebral cortex. 4) Exercise training reduced early aged hypertension hypertension-induced neural Fas/FasL-mediated caspase-dependent apoptotic pathway and the effect was confirmed by the reduction in expression levels of FasL, Fas, TNF- $\alpha$, TNF receptor 1, FADD, active Caspase-8, and active Caspase- 3 in the cerebral cortex. 5) Exercise training attenuated early aged hypertension hypertension-induced neural mitochondria-mediated caspase-dependent apoptotic pathway, as indicated by the decreases in expression levels of Bax, Bak/Bcl-xL, tBid, Apaf-1, Cytochrome c, active Caspase-9, and active Caspase-3 in the cerebral cortex; 7) Exercise training enhanced Bcl-2 familyrelated pro-survival protein levels $(\mathrm{Bcl}-2, \mathrm{Bcl}-\mathrm{xL}, \mathrm{pBad}$, 14-3-3) and IGF-1- related pro-survival protein levels (IGF-1, pPI3K/PI3K, pAKT/AKT) in the early aged hypertensive cerebral cortex. Taking our findings with the previously apoptotic theories together, we drew the hypothesized diagram (Figure 7) which suggested that cerebral cortex EndoG/AIF-related caspaseindependent, Fas/FasL-mediated caspase-dependent and mitochondria-mediated caspase-dependent apoptotic pathways were augmented by early aged hypertension and were attenuated by exercise training. In contrast, the cerebral cortex Bcl-2 family-related and IGF-1-related pro-survival pathways were suppressed by early aged hypertension and were enhanced after exercise training. Exercise training not only has the neuroprotective effects through anti-apoptotic and pro-survival pathways, but also may have other indirect beneficial effects from multiple systems such as enhancing insulin sensitivity, anti-oxidative stress, antiinflammation, hormonal balance, neuromodulatory balance, decreasing neurotoxicity, improving neuronal mitochondrial function or other unclear interaction factors [16].

Hypertension is a devastating vascular risk factor for end-organ damage [34]. Increased apoptosis in hypertensive organs such as the heart (ventricular cardiomyocytes), kidney (inner cortex and medulla), and brain (cortex, striatum, hippocampus, and thalamus) was observed with extensive effect of cell death inducer [35]. Unregulated neural cell death in 
the cerebral cortex could be detrimental as it could deteriorate neural function and this sequester of uncontrolled cell death could be influenced in certain diseases. Hypertension induced a neural loss in the brain which would further aggravate blood flow reduction and impaired function resulting in end-stage brain damage [36]. Evidence revealed that hypertension extended ischemic brain lesions and was progressively observed to coexist with neurodegenerative illness like Alzheimer's Disease [37].
Since hypertension is the main reason for the increase of neural loss and deterioration of brain performance, therefore evaluating the degree of neural apoptosis at early stage of hypertension might be significant to minimize the subsequent complications.

Exercise training is a significant lifestyle modification for improving general body health. The benefit of exercise training in hypertension is determined by changes in the peripheral and central mechanisms of
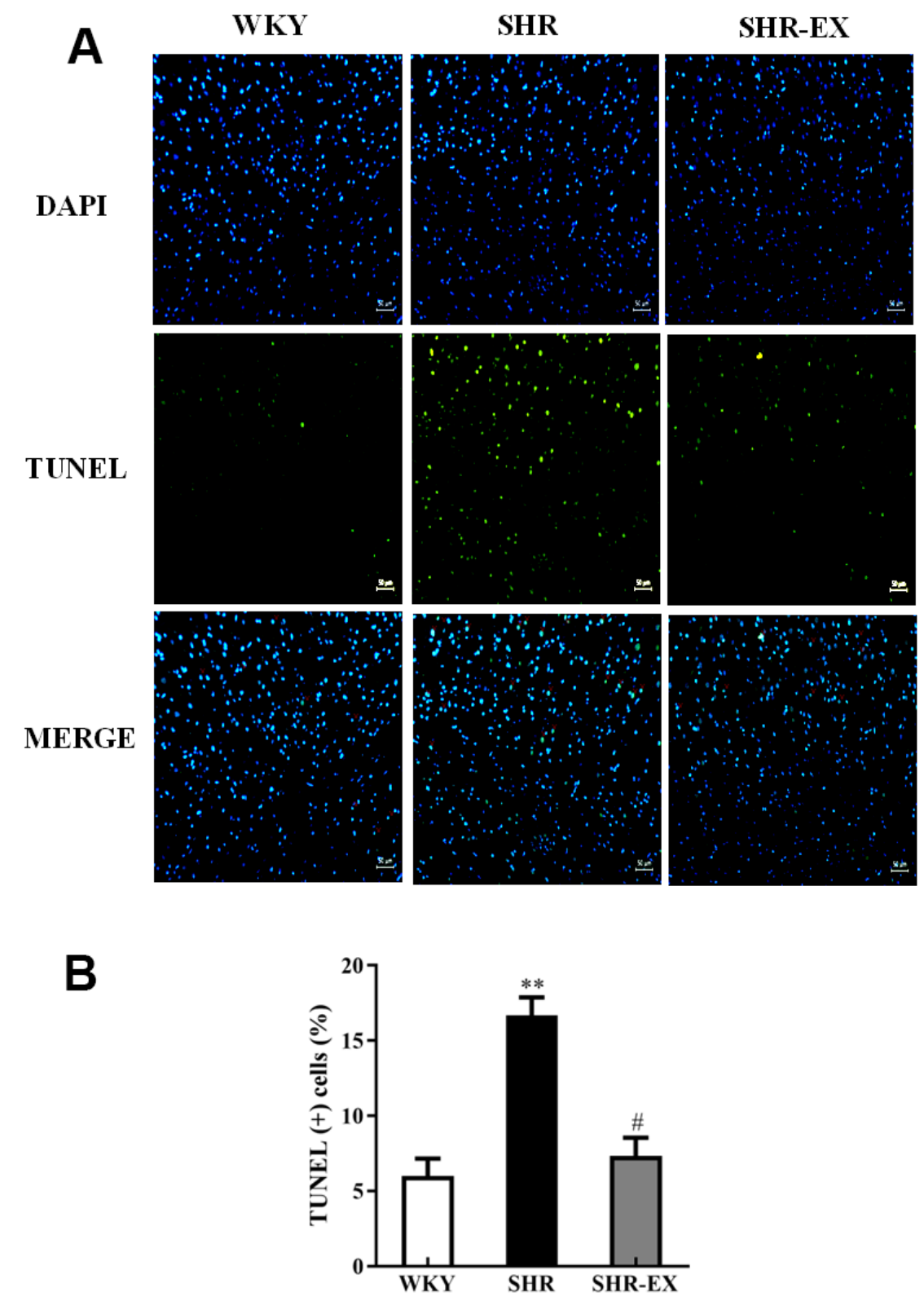

Figure 1. (A) Positive apoptotic neural cell was identified with 4',6-diamidino-2-phenylindole (DAPI) staining and terminal deoxynucleotidyl transferase dUTP nick end labeling (TUNEL) assay. The upper image with blue spots indicates DAPI staining, middle image with green spots indicates TUNEL assay, and lower image with blue and green spots indicates Merge of DAPI staining and TUNEL assay. Scale bar, 50 $\mu m$. (B) The bar shows the quantification of TUNEL apoptosis (\%), determined as the proportion of TUNEL-positive cells relative to total DAPI stained

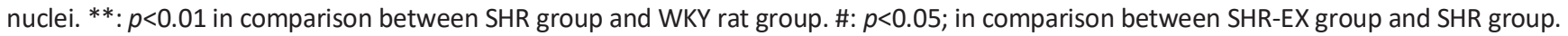


blood pressure control [11]. A previous study showed that low intensity of exercise training substantially decreased systolic blood pressure, with high chances of reducing the diastolic blood pressure in stage onehypertension [38]. In this present study, the systolic blood pressure, diastolic blood pressure and mean blood pressure in early aged hypertensive rats were decreased after 12 weeks of exercise training on a treadmill. Base on the results of this study, we reconfirmed that exercise training could be considered as an alternative or adjunct to treat hypertension as well as an adjuvant to prevent hypertension-induced complications.

Both human and animal studies had substantiated that exercise training could effectively improve brain function and provide neuroprotection [39, 40]. Substantial reduction of AIF expression or suppressing AIF activity provided neuroprotection against intense neurodegeneration [41]. In this present study, exercise training was found to reduce hypertension-induced EndoG/AIF-related caspase-independent apoptotic pathways in the cerebral cortex which was evidenced by reduced expression levels of EndoG and AIF. No previous study had showed exercise training prevents neural EndoG and AIF in hypertensive brain, but one animal study reported that exercise training prevented neural cell death with a significant reduction of apoptosis-inducing factor (AIF) in the brain undergoing ischemia and reperfusion [25]. The suppression of EndoG and AIF protein in the early aged hypertensive cerebral cortex by exercise training was first reported in this study.

Our present study demonstrated that exercise training played a significant role in inhibiting hypertensioninduced Fas/FasL-mediated caspase-dependent apoptotic pathway in the cerebral cortex, as indicated by decreased expression levels of FasL, Fas, FADD, active Caspase-8, and active Caspase-3. A previous study reported that swimming exercise on D-galactoseinduced aging rats decreased protein Fas ligand, Fas, FADD and active Caspase- 8 expression in the hippocampus, which ultimately suppressed apoptosis [33]. Another evidence postulated that significant down-regulation of Fas receptor was neuroprotective and could reduce posttraumatic axonal degeneration after acute spinal injury [42]. Primarily, exercise training decreased activities of Fas/FasL-mediated caspase-dependent apoptotic pathway in the cerebral cortex of early aged hypertensive rat was preliminarily revealed in this study.

Neural mitochondria-mediated caspase-dependent apoptotic pathway responding to apoptosis depends on
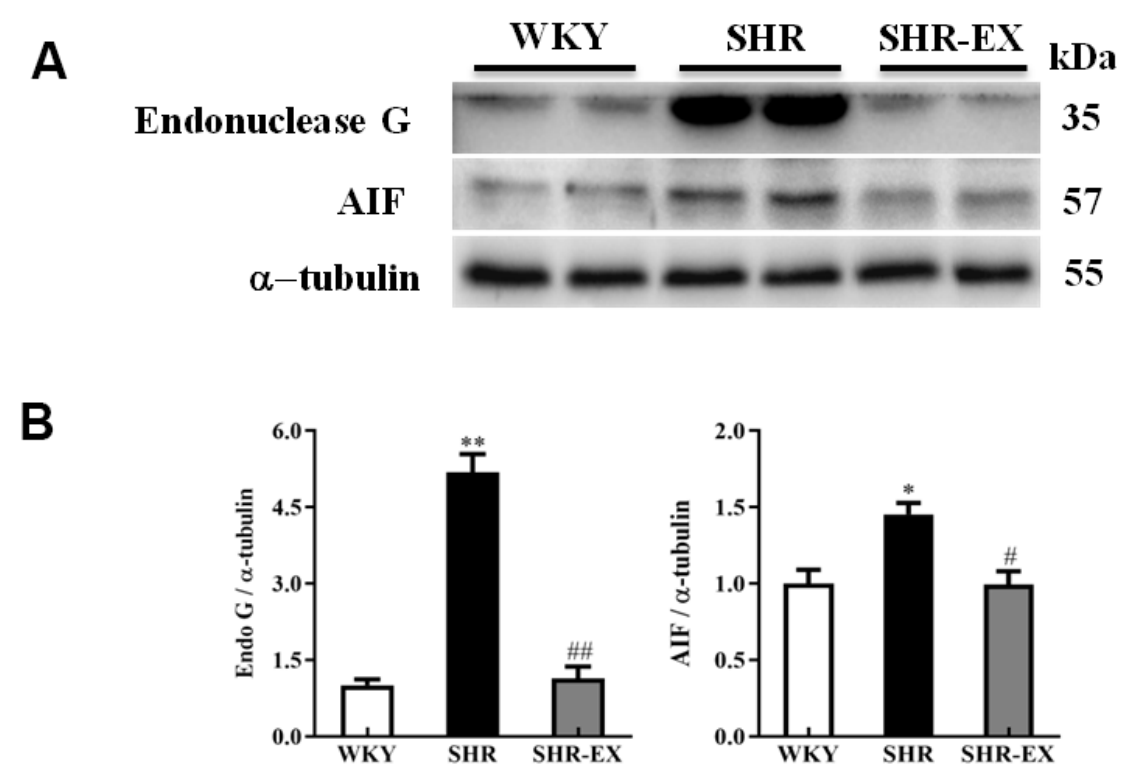

Figure 2. The endonuclease G (EndoG) and apoptosis-inducing factor (AIF) caspase-independent apoptotic pathway in a normotensive Wistar Kyoto (WKY) group, a spontaneously early aged hypertensive (SHR) group and a hypertension with 12 weeks exercise training (SHR-EX) group. (A) The 3 representative protein levels of AIF and EndoG extracted from the cerebral cortex of excised brain in WKY, SHR, and SHR-EX groups were measured by Wester blotting analysis. The $\alpha$-tubulin was used as an internal control. (B) Bars represent the relative fold changes of protein quantification relative to the control group in EndoG and AlF proteins on $\alpha$-tubulin and mean values \pm SD ( $n=6$ in each group). *: $p<0.05,{ }^{* *}: p<0.01$ in comparison between SHR group and WKY rat group; \#: $p<0.05, \# \#: p<0.01$ in comparison between SHR-EX group and SHR group. 
the balance between the pro-apoptotic and anti-apoptotic factors [43]. A previous study revealed that pre-exercise suppressed pro-apoptotic B-cell lymphoma 2 (Bcl-2) family molecules such as Bid in traumatic brain injury, diminished mitochondrial permeabilization with prevented release of Cytochrome c, and decreased migration of apoptosis-inducing factor (AIF) to the nucleus and prevented caspase activation [19]. In this current study, exercise training was found to attenuate hypertension-induced neural mitochondria-mediated caspase-dependent apoptotic pathway. This corroborated finding was based on decreased expression levels of Bax/Bcl-2, Bak/Bcl-xL, tBid, Apaf-1, Cytochrome $c$, active Caspase-9, and active Caspase- 3 in the cerebral cortex after 12 weeks of exercise training. A previous study showed that physical exercise improved brain cortex and cerebellum through reduction of pro-apoptotic Bax/Bcl-2 proportion [16].

The recovery of neurological disorders mainly relies on neural cellular survival [25]. Former research indicated that exercise training simultaneously suppressed cell
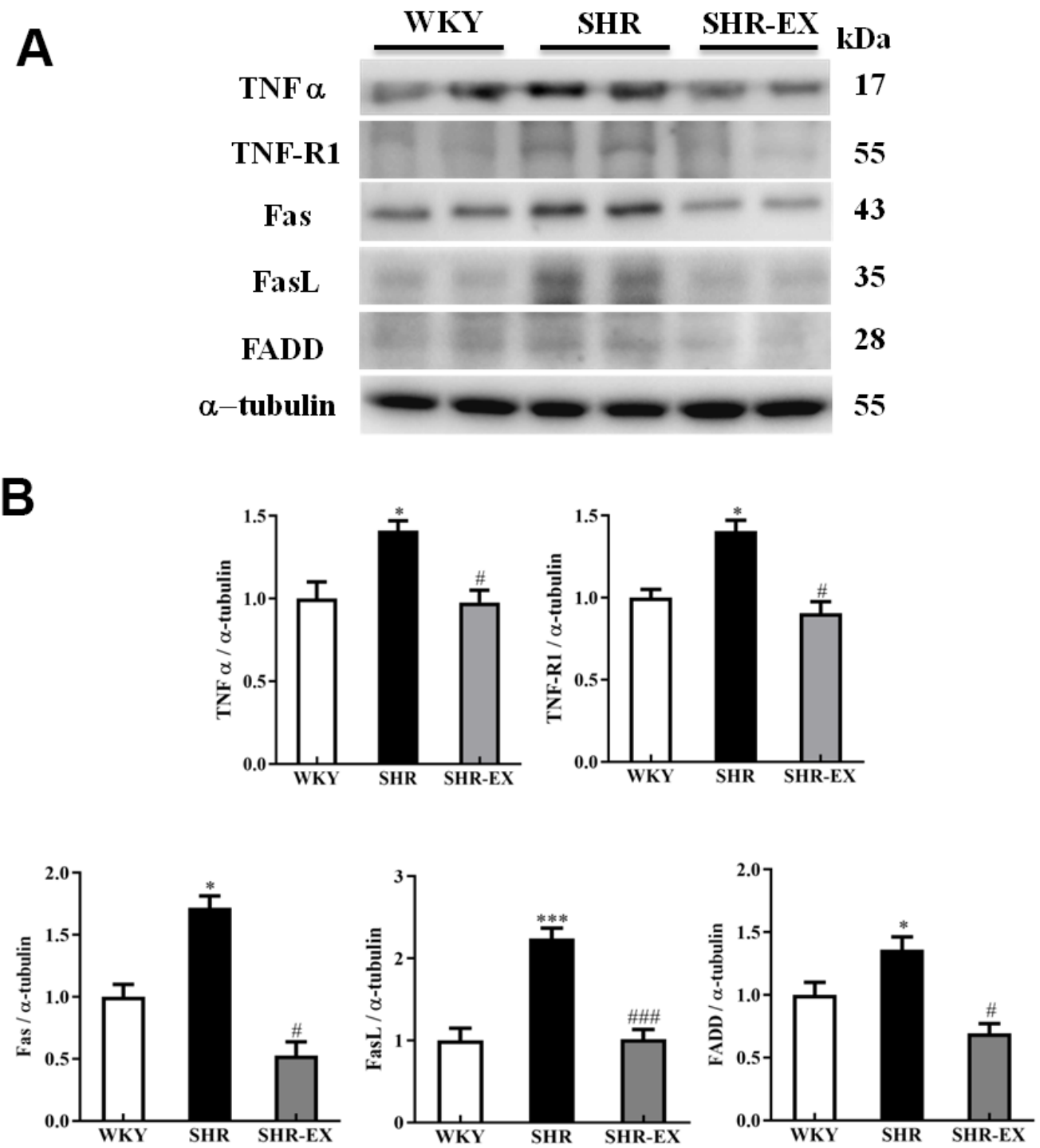

Figure 3. The upstream components of Fas/FasL-mediated caspase-dependent apoptotic pathway in a normotensive Wistar Kyoto (WKY) group, a spontaneously early aged hypertensive (SHR) group and a hypertension with 12 weeks exercise training (SHR-EX) group. (A) The representative protein levels of TNF $\alpha$, TNF-R1, Fas receptor (Fas), Fas ligand (FasL), and Fas-associated death domain (FADD), extracted from the cerebral cortices of excised brain in WKY, SHR, and SHR-EX groups were measured by western blot analysis. The $\alpha$-tubulin was used as an internal control. (B) Bars represent the relative fold changes of protein quantification relative to the control group in TNF $\alpha$, TNF-R1, Fas, FasL, and FADD on $\alpha$-tubulin and mean values \pm SD ( $n=6$ in each group). ${ }^{*}: p<0.05,{ }^{* * *}: p<0.001$ in comparison between SHR group and WKY rat group. \#: $p<0.05$; \#\#\#: $p<0.001$, in comparison between SHR-EX group and SHR group. 


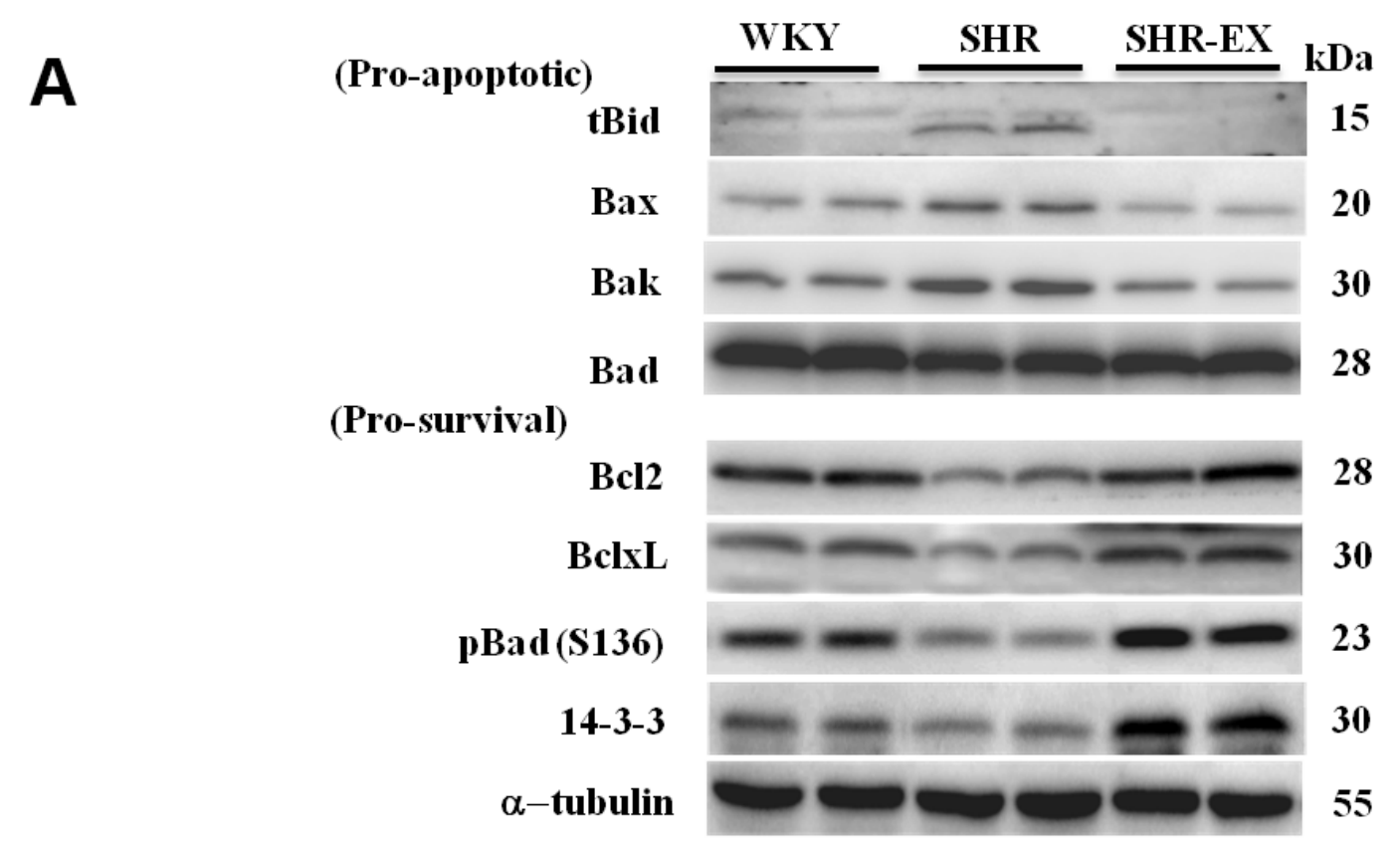

\section{B Pro-apoptotic}
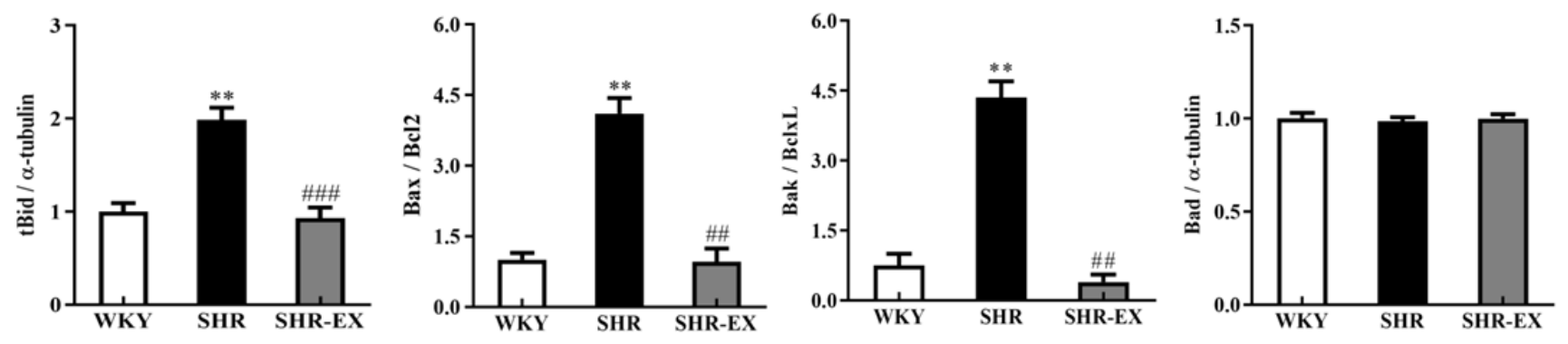

\section{Pro-survival}
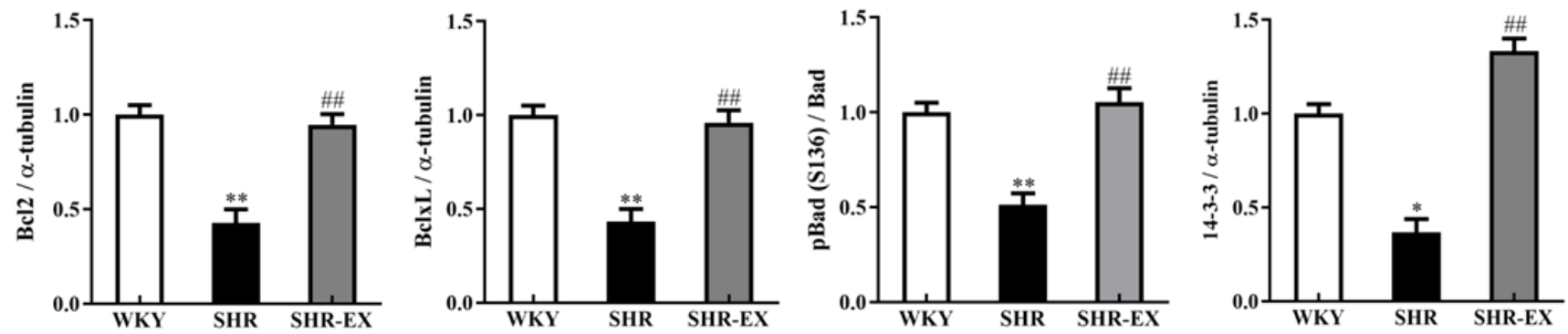

Figure 4. The upstream components of mitochondria-mediated caspase-dependent apoptotic pathway in a normotensive Wistar Kyoto (WKY) group, a spontaneously early aged hypertensive (SHR) group and a hypertension with 12 weeks exercise training (SHR-EX) group. (A) The representative protein levels of $\mathrm{t}-\mathrm{Bid}$ (BH3 interacting domain death agonist), Bax (Bcl-2 Associated X), Bak (Bcl-2-agonist/killer 1), Bad (Bcl-2 agonist cell death) as well as Bcl-2, Bcl-xL, p-Bad, and 14-3-3 proteins extracted from western blotting analysis. The $\alpha$-tubulin was used as an internal control. (B, C) Bars represent the cerebral cortices of excised brain in WKY, SHR, and SHR-EX groups were measured by $\mathrm{Wt}$ the pro-apoptotic as well as pro-survival relative fold changes of protein quantification relative to the control group in t-Bid, Bax/Bcl-2, Bak/Bcl-xL as well as pBad/Bad pBad and 14-3-3 on $\alpha$-tubulin, respectively, and mean values \pm SD ( $n=6$ in each group). *: $p<0.05,{ }^{* *}: p<0.01$, in comparison between SHR and WKY rat group; \#\#: $p<0.01, \# \# \#$ : $p<0.001$ in comparison between SHR-EX group and SHR group. 


\section{Downstream}

A

(Fas)

Active Caspase-8

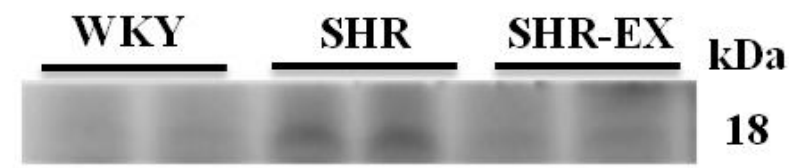

(Mitochondria )

Cytochrome $C$

Apaf-1

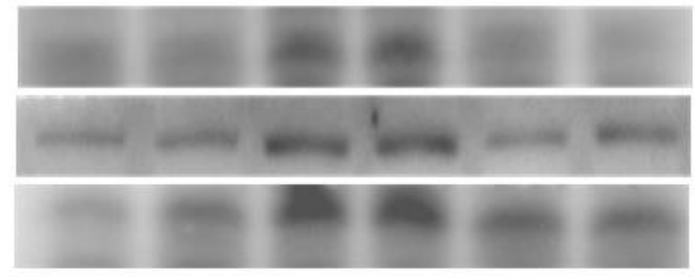

14

135

Active Caspase-9

(Fas-Mitochondria)

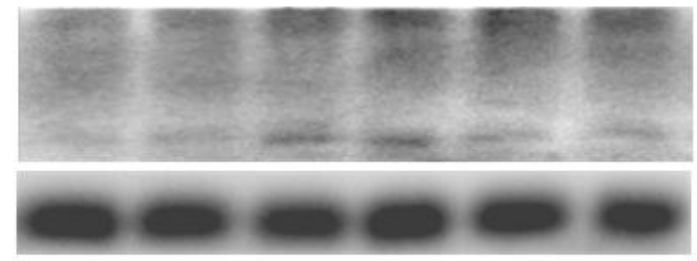

Active Caspase-3

$\alpha$-tubulin

B
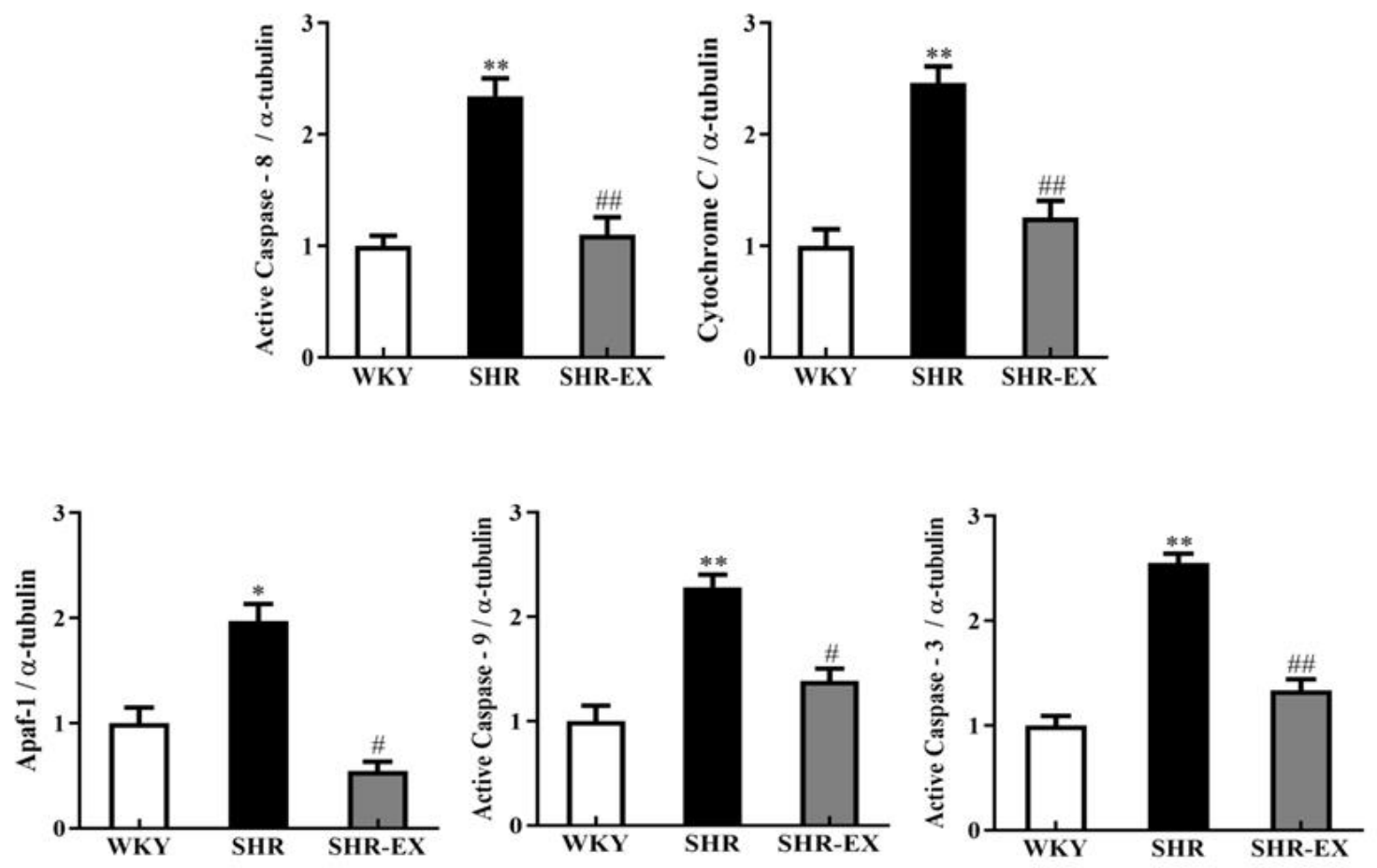

Figure 5. The downstream components of Fas/FasL-mediated and mitochondria-mediated caspase-dependent apoptotic pathways in a normotensive Wistar Kyoto (WKY) group, a spontaneously early aged hypertensive (SHR) group and a hypertension with 12 weeks exercise training (SHR-EX) group. (A) The representative protein levels of active Caspase-8 (Fas downstream), Cytochrome $c$, Apaf-1, active Caspase-9 (mitochondrial downstream), active Caspase-3 (Fas and Mitochondrial downstream) extracted from the cerebral cortex of excised brain in WKY, SHR, and SHR-EX groups were measured by Wester blotting analysis. The $\alpha$ tubulin was used as an internal control. (B) Bars represent the relative fold changes of protein quantification relative to the control group in active Caspase-8, Cytochrome $C$, Apaf-1, active Caspase-9, active Caspase-3 on $\alpha$-tubulin and mean values \pm SD ( $n=6$ in each group). *: $p<0.05, * *: p<0.01$ in comparison between SHR group and WKY rat group; \#: $p<0.05, \# \#<0.01$ in comparison between SHR-EX group and SHR group. 
death pathways and enhanced survival pathways in brain subjected with ischemia/reperfusion injury as well as increased expression of Bcl-xL proteins [25]. Increased anti-apoptotic activities reduced neuronal loss in brain ischemia [44]. Interestingly, in this investigation, exercise training was observed to enhance Bcl-2 family-related pro-survival protein levels (Bcl-2, Bcl-xL, pBad, 14-3-3) and IGF-1-related pro-survival protein levels (IGF-1, pPI3K/PI3K, pAKT, AKT) in the early aged hypertensive cerebral cortex, suggesting another possible therapeutic approach. The increased expression of pPI3K, pAKT and $\mathrm{Bcl}-\mathrm{xL}$ in the hippocampus of aging rats undergoing swimming was found essential to prevent neural apoptosis [33]. Four weeks of moderate-intensity exercise training on aging mice was found to induce 14-3-3 protein, heat shock proteins 70 and increase related neurogenesis biomarkers in the hippocampus with significant effect of enhancing neuroprotection, neurogenesis and synaptic strength [45]. Moreover, exercise training increased optimal blood flow and activated growth factors in traumatic brain injury and improved brain function via promoting neurogenesis, angiogenesis and synaptogenesis [46]. The effects of exercise training on Bcl-2 familyrelated pro-survival protein and IGF-1-related prosurvival protein in the early aged hypertensive cerebral cortex were first investigated in this study.

\section{CONCLUSION}

This present study showed that twelve weeks of exercise training prevented neural apoptosis in early aged hypertensive rat cerebral cortex by suppressing the activation of pro-apoptotic pathways and enhancing pro-survival pathways. Based on the former studies, there was no similar study to support the recent finding in early aged hypertensive rat cerebral cortex. The results of this study suggested that exercise training might have significant benefits on the brain. In general, the study hereby supported the therapeutic abstract to advance the significance of exercise training for treating and preventing neurological abnormalities associated with hypertension.

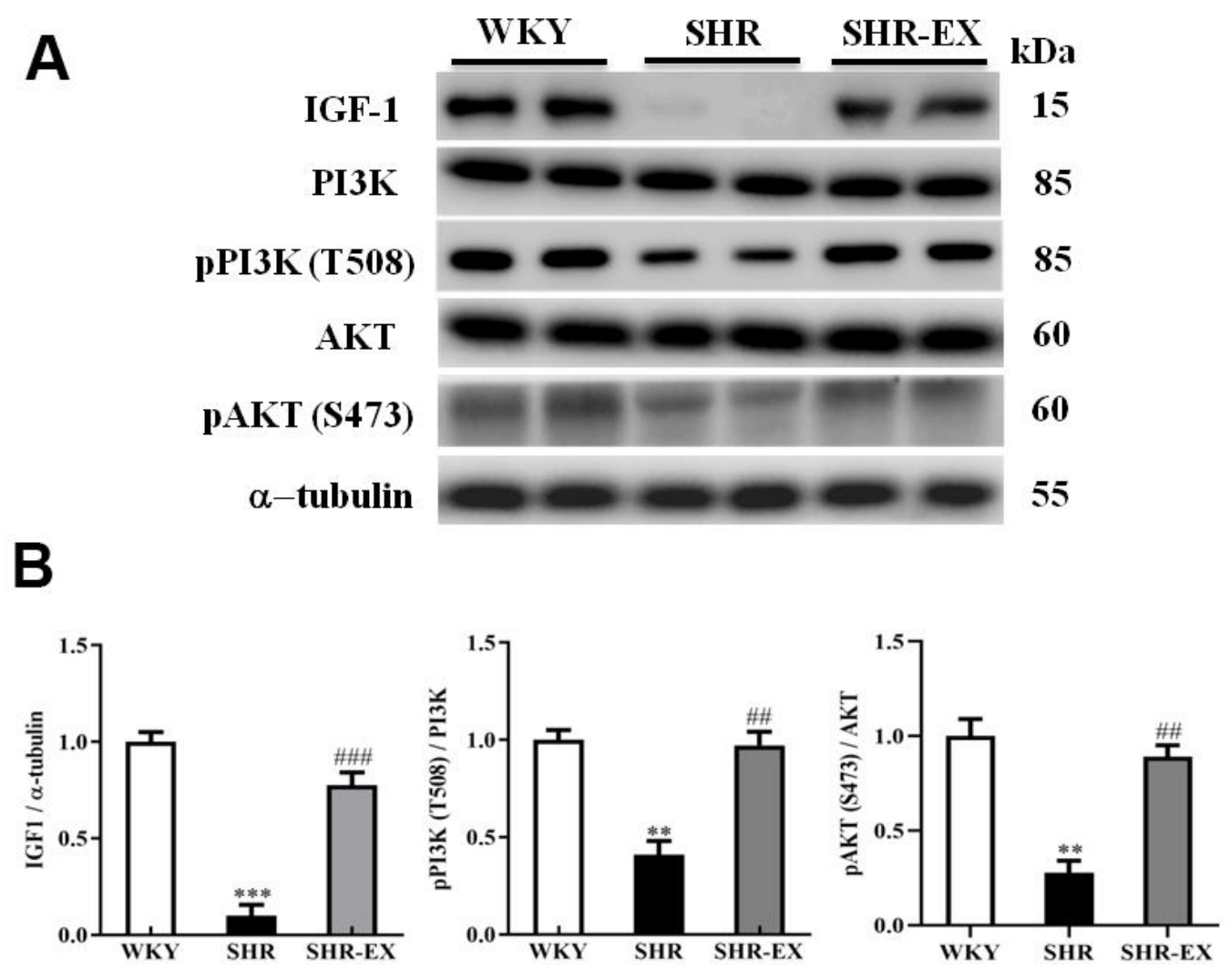

Figure 6. The IGF-1-related survival pathway in a normotensive Wistar Kyoto (WKY) group, a spontaneously early aged hypertensive (SHR) group and a hypertension with 12 weeks exercise training (SHR-EX) group. (A) The representative protein levels of IGF-1, PI3K, p-PI3K, AKT and p-AKT protein extracted from the cerebral cortex of excised brain in WKY, SHR, and SHR-EX groups were measured by western blot analysis. The $\alpha$-tubulin was used as an internal control. (B) Bars represent the relative changes of protein quantification in IGF-1, p-PI3K/PI3K, and pAKT/AKT on $\alpha$-tubulin and mean values \pm SD ( $n=6$ in each group). ${ }^{* *}: p<0.01, * * *: p<0.001$, in comparison between SHR group and WKY rat group; \#\#: $p<0.01$, \#\#\#: $p<0.001$, in comparison between SHR-EX group and SHR group. 


\section{Perspectives}

Hypertension is regarded as a critical vascular risk factor for developing cognitive impairment and brain damage. A disease modifying approach for the treatment and prevention of abnormal brain function in hypertension is desperately needed. Apart from antihypertensive medications, exercise training would be considered as the most rational approach for enhancing neuroprotection and neurorestoration of hypertension. Base on the result of this study, hypertension was known to induce neural apoptosis in

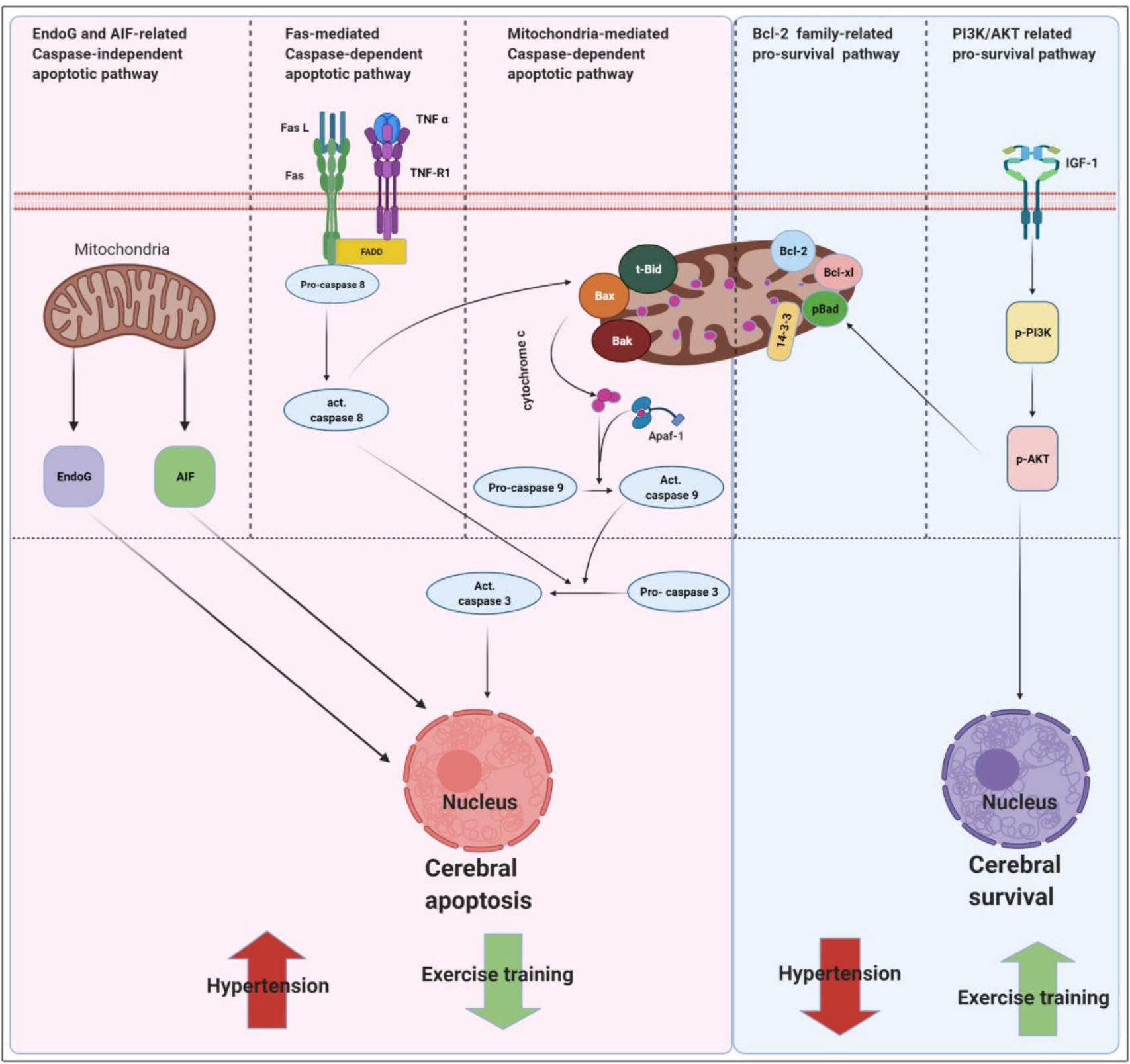

Figure 7. Proposed hypothesis indicating that early aged hypertension appear to activate the EndoG/AIF-related caspaseindependent, Fas/FasL-mediated caspase-dependent apoptotic pathways (Fas ligand, Fas receptor, TNF- $\alpha$, TNF receptor 1, Fas-associated death domain, active Caspase-8 and active Caspase-3) and mitochondria-mediated caspase-dependent apoptotic pathway (t-Bid, Bax, Bak, Bad, cytochrome c, Apaf-1, active Caspase 9 and active Caspase-3) as well as suppresses Bcl-2 family-related pro-survival pathway (Bcl2, Bcl-xL, p-Bad, 14-3-3) and IGF-1 related pro-survival pathway (IGF-1, pPI3K/PI3K, and pAKT/AKT). Whereas, exercise training tends to inhibits early aged hypertension-induce neural EndoG/AIF related caspase-independent, hypertension-induce Fas/FasL-mediated apoptotic and hypertension-induce mitochondria-mediated caspasedependent apoptotic pathway as well as enhances Bcl-2 family-related pro-survival and IGF-1-related pro-survival pathway. 
the cerebral cortex and was attenuated by exercise training. Therefore, it is essential to realize the possibility for generating brain abnormalities in aged hypertension as well as the significant of exercise training as a therapeutic approach for preventing brain degeneration in aged hypertension. Since it is strenuous to extract cerebral tissues from human brain, this current animal demonstration might help to provide a panorama and elucidation of the mechanism on how exercise training attenuates brain damage or neural apoptosis related to neurological disorders in human. The study explicates the effect of 12 weeks of exercise training against early aged hypertension-induce neural apoptosis in the cerebral cortex through suppressing pro-apoptotic pathways and activating anti-apoptotic activities as well as enhancing the pro-survival pathway, which may aid in preventing other neurological conditions resulting in brain damage. Further clinical studies are needed to elucidate any feasible therapeutic approach in human.

\section{MATERIALS AND METHODS}

\begin{abstract}
Animal
The experiment was performed on normotensive Wistar Kyoto rats (WKY, $\mathrm{n}=10)$ and spontaneously early aged hypertensive male rats (SHR, $\mathrm{n}=20)$ at 12 months old, males. The spontaneously early aged hypertensive rats (SHR) were further divided equally into sedentary (SHR) group and exercise (SHR-EX) group. The rats were housed under a 12-h light-dark cycle to maintain good living conditions. Standard laboratory chow (Lab Diet 5001; PMI Nutrition International, Brentwood, MO, USA) and water ad libitum were used for feeding the rats. The protocol applied for the animal caring was following The National Institutes of Health Guide for the Care and Use of Laboratory Animals and approved by the committee of China Medical University Animal Center, Taichung.
\end{abstract}

\section{Exercise training}

A motor-driven levelled treadmill (Model T408E, Diagnostic and Research Instruments Co., Taoyuan, Taiwan) was used for animal exercise. On the first day, only SHR-EX group was prepared to run at $15 \mathrm{~m} / \mathrm{min}$ for $20 \mathrm{~min}$ in order to develop habitual acquaintance with the training. The running period was gradually increased by 10 min each day until it reached a running period of $60 \mathrm{~min} /$ day within five days for which habitual acquaintance of training was achieved. During the training period, we placed all the three groups on the treadmill. The SHR-EX group run at a speed of $18 \mathrm{~m} / \mathrm{min}$ then gradually accelerated at a speed of 3 $\mathrm{m} / \mathrm{min}$ in every 2 weeks up to $27 \mathrm{~m} / \mathrm{min}$. The SHR-EX group was trained at $60 \mathrm{~min}$ in each day for 5 days a week totaling for 12 weeks. Whereas, the other groups (WKY and SHR) were assigned to run without undergoing similar environmental stimulation as SHREX group.

\section{Measurement of blood pressure}

At rest, systolic, diastolic and mean arterial blood pressure of the animals were measured applying the Indirect Tail cuff method (BP98A, Softron, Tokyo, Japan) once a week before conducting the sacrifice.

\section{Citrate synthase activity}

The effectiveness of exercise training was confirmed by increased in Citrate Synthase Activity. We measured the Citrate Synthase Activity by using homogenized soleus muscle samples (in five volumes of 0.1 Mof Tris buffer containing $0.1 \%$ Triton $\mathrm{X}-100$ ) from all the three groups (WKY, SHR and SHR-EX). We used spectrophotometric readings at $412 \mathrm{~nm}$ (UV-240, Shimadzu Co., Tokyo, Japan). All samples were tested in duplicate.

\section{Preparation of brain tissue}

The rats were first put under anaesthesia to minimize suffering. Isoflurane solution (2\% concentration) delivered through carbogen gas $\left(95 \% \mathrm{O}_{2}\right.$ and $\left.5 \% \mathrm{CO}_{2}\right)$ was used for anesthetizing the rats by inhalation. After being sacrificed, the brain tissues were removed and cleaned with cold ice of $0.9 \%$ Sodium chloride $(\mathrm{NaCl})$ and weighed, then placed in a $4 \%(\mathrm{w} / \mathrm{v})$ solution of PFA for $15 \mathrm{~min}$, transferred to a $20 \%$ (w/v) fresh sucrose solution for 12-16 hours, and then retransferred to a $30 \%(\mathrm{w} / \mathrm{v})$ fresh sucrose solution for 12-16 hours. The brain tissues were sliced into the cerebral cortex section and tested by DAPI /TUNEL staining and western blot.

\section{TUNEL assay and DAPI staining}

After the decapitation, the cut brain pieces were soaked in neutral buffered formalin (NBF) then deparaffinized with Xylene. Graded alcohols were used to rehydrate the deparaffinized sections. After being soaked in 200 $\mathrm{ml} 0.1 \mathrm{M}$ Citrate buffer ( $\mathrm{pH} 6$, with the $750 \mathrm{~W}$ (high) microwave irradiation) for $1 \mathrm{~min}$, the sections were blocked in the buffer for $30 \mathrm{~min}$ and washed twice in PBS. The tissue sections were mounted for TUNEL assay (Terminal deoxynucleotidyl transferase and fluorescein isothiocyanate-dUTP assay) using an apoptosis detection kits (Roche Applied Science, Indianapolis, IN, USA) for $60 \mathrm{~min}, 37^{\circ} \mathrm{C}$ as well as adding DAPI Fluoromount $\mathrm{G}$ for $15 \mathrm{~min}$ at $18-25^{\circ} \mathrm{C}$. For fluorescence evaluation by using an Inverted 
Microscope (Eclipse Ti-U, NIKON, Japan), the excitation wavelength in the range of $450-500 \mathrm{~nm}$ and detection in the range of 515-565 nm (green) and 358461 (blue) were used. We determined the number of TUNEL-positive cells and the DAPI-stained nuclei on different separated fields (like 3 fields/sample and 3 samples/group) of the cerebral cortex in random selections and repeated the process for at least nine times. To avoid bias and ensure all results are reliable, two independent individuals conducted the counting separately.

\section{Electrophoresis and western blot}

Bradford method (Bio-Rad Protein Assay, Hercules, CA, USA) was applied to measure the protein concentration of neural tissues obtained from the cerebral cortex. Protein samples $(100 \mu \mathrm{g} / \mathrm{lane})$ were divided on a $12 \%$ SDS-PAGE (sodium dodecyl sulfatepolyacrylamide gel electrophoresis) at a voltage of $80 \mathrm{~V}$ for 20 minutes then at $100 \mathrm{~V}$ for 2 hours. After the electrophoresis, proteins tissues were transferred to polyvinylidene difluoride (PVDF) membranes (Millipore, Bedford, MA, USA) at a constant voltage of $100 \mathrm{~V}$ for 1.5 hours. $5 \%$ of non-fat milk was used for one hour to block PVDF membranes. The membranes were incubated overnight at $4^{\circ} \mathrm{C}$ with a diluted solution mixed with primary antibodies including IGF-1, AKT, pAKT (S473), PI3K, Bcl-2, Bcl-xL, pBad (S136), Bad, Bax, Bak, Cytochrome $c$, TNF $\alpha$, TNF-R1, FasL, Fas receptor, FADD, Apaf-1, active Caspase-8, active Caspase-9, active Caspase-3 with 1:1000 dilution (Cell Signalling Technology, Beverly, MA, USA) and 14-3-3, pPI3K (T508), EndoG, AIF, $\alpha$-tubulin with 1:500 dilution (Santa Cruz Biotechnology, Santa Cruz, CA) were incubated in antibody binding buffer at $4^{\circ} \mathrm{C}$ overnight, washed by TBST, incubated with an HRPconjugated second antibody solution diluted at 1:5000 (Santa Cruz Biotechnology, Santa Cruz, CA, USA) at room temperature, and then washed in the TBST buffer. With an enhanced chemiluminescence (ECL) Western blot reagent (Millipore Corporation, Bedford, MA), the immunoblotted proteins were visualized and quantified by a chemiluminescence detection system (Fujifilm LAS-3000, Fuji, Tokyo, Japan). The density of the bands was quantified by densitometry using Gel-Pro Analyzer densitometry software (Media Cybernetics, Silver Spring, MD, USA).

\section{Statistical analysis}

All data including brain weights, level of proteins, and percentage of TUNEL-positive cells were compared among control (WKY), sedentary (SHR) and exercise (SHR-EX) group, respectively. One-way analysis of variance (one-way ANOVA) statistical technique was applied to perform pre-planned contrast comparison for negative or positive control. SPSS 22.0 software was used for analyzing and $p<0.05$ was regarded as significant.

\section{AUTHOR CONTRIBUTIONS}

Yijie Liu, Zhen-Yang Cui, contributed equally to this work. Chun-lei Shan, and Shin-Da Lee contributed equally to this work as senior authors. Yijie Liu, ZhenYang Cui, Chun-lei Shan, Shin-Da Lee contributed to the conceptualization; Yijie Liu, Ai-Lun Yang, Chun-lei Shan, and Shin-Da Lee contributed methodologyexperimental resources; Yijie Liu, Amadou W. Jallow, Hai-Liang Huang, and Ai-Lun Yang performed Methodology; Yijie Liu and Shin-Da Lee contributed Data curation; Amadou W. Jallow and Shin-Da Leecontributed original draft; Yijie Liu, Zhen-Yang Cui Chun-lei Shan, Shin-Da Leecontributed editing and revising the manuscript. All authors approved the final version of the manuscript.

\section{CONFLICTS OF INTEREST}

The authors declare that they have no conflicts of interest.

\section{FUNDING}

This study was partially supported by the Taiwan Ministry of Science and Technology (MOST 107-2314B-468-002-MY3). This study was supported by grants through (Shanghai Health Commission) Accelerated the Development of Traditional Chinese Medicine ThreeYear Action Plan Project (of Shanghai Health Commission) [Grant No.: ZY(2018-2020)-CCCX-200106; ZY(2018-2020)-CCCX-2004-05]. This study was partially supported by Weifang Medical University and Shanghai University of Traditional Chinese Medicine. The diagram of Figure 7 was created with BioRender.com. Our gratitude goes to Michael Burton for proofreading, Asia University.

\section{REFERENCES}

1. Oparil S, Acelajado MC, Bakris GL, Berlowitz DR, Cífková R, Dominiczak AF, Grassi G, Jordan J, Poulter NR, Rodgers A, Whelton PK. Hypertension. Nat Rev Dis Primers. 2018; 4:18014. https://doi.org/10.1038/nrdp.2018.14 PMID:29565029

2. Smith JA, Raisky J, Ratliff SM, Liu J, Kardia SL, Turner ST, Mosley TH, Zhao W. Intrinsic and extrinsic epigenetic age acceleration are associated with hypertensive target organ damage in older African Americans. BMC Med Genomics. 2019; 12:141. 
https://doi.org/10.1186/s12920-019-0585-5 PMID:31640709

3. Faraco G, ladecola C. Hypertension: a harbinger of stroke and dementia. Hypertension. 2013; 62:810-17. https://doi.org/10.1161/HYPERTENSIONAHA.113.0106 3 PMID:23980072

4. Jeon SY, Byun MS, Yi D, Lee JH, Choe YM, Ko K, Sohn BK, Choi HJ, Lee JY, Lee DY, and KBASE Research Group. Influence of hypertension on brain amyloid deposition and Alzheimer's disease signature neurodegeneration. Neurobiol Aging. 2019; 75:62-70.

https://doi.org/10.1016/j.neurobiolaging.2018.11.001 PMID:30553154

5. Gabin JM, Tambs K, Saltvedt I, Sund E, Holmen J. Association between blood pressure and Alzheimer disease measured up to 27 years prior to diagnosis: the HUNT Study. Alzheimers Res Ther. 2017; 9:37. https://doi.org/10.1186/s13195-017-0262-x PMID:28569205

6. Gu X, Cai Z, Cai M, Liu K, Liu D, Zhang Q, Tan J, Ma Q. Protective effect of paeoniflorin on inflammation and apoptosis in the cerebral cortex of a transgenic mouse model of Alzheimer's disease. Mol Med Rep. 2016; 13:2247-52.

https://doi.org/10.3892/mmr.2016.4805

PMID:26796245

7. Haspula D, Clark MA. Neuroinflammation and sympathetic overactivity: Mechanisms and implications in hypertension. Auton Neurosci. 2018; 210:10-17.

https://doi.org/10.1016/j.autneu.2018.01.002 PMID:29361405

8. Zhang K, Yang S, Luo C. TNF-alpha and TNF-R1 regulate bupivacaine-induced apoptosis in spinal cord dorsal root ganglion neuron. Eur J Pharmacol. 2018; 833:63-68.

https://doi.org/10.1016/i.ejphar.2018.05.034 PMID:29802833

9. Brown RC, Davis TP. Calcium modulation of adherens and tight junction function: a potential mechanism for blood-brain barrier disruption after stroke. Stroke. 2002; 33:1706-11.

https://doi.org/10.1161/01.str.0000016405.06729.83 PMID:12053015

10. Gąsecki D, Kwarciany M, Nyka W, Narkiewicz K. Hypertension, brain damage and cognitive decline. Curr Hypertens Rep. 2013; 15:547-58. https://doi.org/10.1007/s11906-013-0398-4 PMID:24146223

11. Wang T, Maltez MT, Lee HW, Ahmad M, Wang HW, Leenen $\mathrm{FH}$. Effect of exercise training on the FNDC5/BDNF pathway in spontaneously hypertensive rats. Physiol Rep. 2019; 7:e14323. https://doi.org/10.14814/phy2.14323

PMID:31883222

12. Sabbahi A, Arena R, Elokda A, Phillips SA. Exercise and Hypertension: Uncovering the Mechanisms of Vascular Control. Prog Cardiovasc Dis. 2016; 59:226-34.

https://doi.org/10.1016/j.pcad.2016.09.006 PMID:27697533

13. Ding $Y$, Li J, Luan $X$, Ding $Y H$, Lai $Q$, Rafols JA, Phillis JW, Clark JC, Diaz FG. Exercise pre-conditioning reduces brain damage in ischemic rats that may be associated with regional angiogenesis and cellular overexpression of neurotrophin. Neuroscience. 2004; 124:583-91.

https://doi.org/10.1016/j.neuroscience.2003.12.029 PMID:14980729

14. Vecchio LM, Meng Y, Xhima K, Lipsman N, Hamani C, Aubert I. The Neuroprotective Effects of Exercise: Maintaining a Healthy Brain Throughout Aging. Brain Plast. 2018; 4:17-52. https://doi.org/10.3233/BPL-180069 PMID:30564545

15. Lautenschlager NT, Cox KL, Flicker L, Foster JK, van Bockxmeer FM, Xiao J, Greenop KR, Almeida OP. Effect of physical activity on cognitive function in older adults at risk for Alzheimer disease: a randomized trial. JAMA. 2008; 300:1027-37.

https://doi.org/10.1001/jama.300.9.1027 PMID: 18768414

16. Marques-Aleixo I, Santos-Alves E, Balça MM, Rizo-Roca D, Moreira PI, Oliveira PJ, Magalhães J, Ascensão A. Physical exercise improves brain cortex and cerebellum mitochondrial bioenergetics and alters apoptotic, dynamic and auto(mito)phagy markers. Neuroscience. 2015; 301:480-95.

https://doi.org/10.1016/j.neuroscience.2015.06.027 PMID:26116519

17. Navarro A, Gomez C, López-Cepero JM, Boveris A. Beneficial effects of moderate exercise on mice aging: survival, behavior, oxidative stress, and mitochondrial electron transfer. Am J Physiol Regul Integr Comp Physiol. 2004; 286:R505-11.

https://doi.org/10.1152/ajpregu.00208.2003 PMID: 14615275

18. Song SH, Jee YS, Ko IG, Lee SW, Sim YJ, Kim DY, Lee SJ, Cho YS. Treadmill exercise and wheel exercise improve motor function by suppressing apoptotic neuronal cell death in brain inflammation rats. J Exerc Rehabil. 2018; 14:911-19.

https://doi.org/10.12965/jer.1836508.254 PMID: 30656148

19. Zhao Z, Sabirzhanov B, Wu J, Faden Al, Stoica BA. Voluntary Exercise Preconditioning Activates Multiple Antiapoptotic Mechanisms and Improves Neurological Recovery after Experimental Traumatic Brain Injury. J Neurotrauma. 2015; 32:1347-60. 
https://doi.org/10.1089/neu.2014.3739

PMID:25419789

20. Vivar $C$, Potter $M C$, van Praag $H$. All about running: synaptic plasticity, growth factors and adult hippocampal neurogenesis. Curr Top Behav Neurosci. 2013; 15:189-210.

https://doi.org/10.1007/7854 2012 220

PMID:22847651

21. Marlatt MW, Potter MC, Lucassen PJ, van Praag $\mathrm{H}$. Running throughout middle-age improves memory function, hippocampal neurogenesis, and BDNF levels in female C57BL/6J mice. Dev Neurobiol. 2012; 72:943-52.

https://doi.org/10.1002/dneu.22009 PMID:22252978

22. Yuan J, Yankner BA. Apoptosis in the nervous system. Nature. 2000; 407:802-09.

https://doi.org/10.1038/35037739 PMID:11048732

23. Büttner S, Eisenberg T, Carmona-Gutierrez D, Ruli D, Knauer $\mathrm{H}$, Ruckenstuhl C, Sigrist C, Wissing S, Kollroser M, Fröhlich KU, Sigrist S, Madeo F. Endonuclease G regulates budding yeast life and death. Mol Cell. 2007; 25:233-46.

https://doi.org/10.1016/j.molcel.2006.12.021 PMID:17244531

24. Candé C, Cohen I, Daugas E, Ravagnan L, Larochette N, Zamzami N, Kroemer G. Apoptosis-inducing factor (AIF): a novel caspase-independent death effector released from mitochondria. Biochimie. 2002; 84: 215-22.

https://doi.org/10.1016/s0300-9084(02)01374-3 PMID:12022952

25. Liebelt B, Papapetrou P, Ali A, Guo M, Ji X, Peng C, Rogers $R$, Curry A, Jimenez D, Ding $Y$. Exercise preconditioning reduces neuronal apoptosis in stroke by up-regulating heat shock protein-70 (heat shock protein-72) and extracellular-signal-regulated-kinase 1/2. Neuroscience. 2010; 166:1091-100.

https://doi.org/10.1016/j.neuroscience.2009.12.067 PMID:20083167

26. Clarke P, Beckham JD, Leser JS, Hoyt CC, Tyler KL. Fasmediated apoptotic signaling in the mouse brain following reovirus infection. J Virol. 2009; 83:6161-70. https://doi.org/10.1128/JVI.02488-08 PMID:19321603

27. Morrison RS, Kinoshita Y, Johnson MD, Ghatan S, Ho JT, Garden G. Neuronal survival and cell death signaling pathways. Adv Exp Med Biol. 2002; 513:41-86. https://doi.org/10.1007/978-1-4615-0123-7 2 PMID: 12575817

28. Kristiansen M, Ham J. Programmed cell death during neuronal development: the sympathetic neuron model. Cell Death Differ. 2014; 21:1025-35.

https://doi.org/10.1038/cdd.2014.47 PMID:24769728
29. Chipuk JE, Green DR. How do BCL-2 proteins induce mitochondrial outer membrane permeabilization? Trends Cell Biol. 2008; 18:157-64.

https://doi.org/10.1016/i.tcb.2008.01.007 PMID:18314333

30. Huat TJ, Khan AA, Pati S, Mustafa Z, Abdullah JM, Jaafar H. IGF-1 enhances cell proliferation and survival during early differentiation of mesenchymal stem cells to neural progenitor-like cells. BMC Neurosci. 2014; 15:91.

https://doi.org/10.1186/1471-2202-15-91 PMID:25047045

31. Ma K, Xu H, Zhang J, Zhao F, Liang H, Sun H, Li P, Zhang $S$, Wang $R$, Chen $X$. Insulin-like growth factor-1 enhances neuroprotective effects of neural stem cell exosomes after spinal cord injury via an miR-219a-23p/YY1 mechanism. Aging (Albany NY). 2019; 11:12278-94.

https://doi.org/10.18632/aging.102568

PMID:31848325

32. Zhao B, Zheng Z. Insulin Growth Factor 1 Protects Neural Stem Cells Against Apoptosis Induced by Hypoxia Through Akt/Mitogen-Activated Protein Kinase/Extracellular Signal-Regulated Kinase (Akt/MAPK/ERK) Pathway in Hypoxia-Ishchemic Encephalopathy. Med Sci Monit. 2017; 23:1872-79.

https://doi.org/10.12659/msm.901055

PMID:28420864

33. Lin JY, Kuo WW, Baskaran R, Kuo CH, Chen YA, Chen WS, Ho TJ, Day $\mathrm{CH}$, Mahalakshmi B, Huang CY. Swimming exercise stimulates IGF1/ PI3K/Akt and AMPK/SIRT1/PGC1 $\alpha$ survival signaling to suppress apoptosis and inflammation in aging hippocampus. Aging (Albany NY). 2020; 12:6852-64.

https://doi.org/10.18632/aging.103046

PMID:32320382

34. Schmieder RE. End organ damage in hypertension. Dtsch Arztebl Int. 2010; 107:866-73. https://doi.org/10.3238/arztebl.2010.0866 PMID:21191547

35. Hamet $P$, Richard L, Dam TV, Teiger E, Orlov SN, Gaboury L, Gossard F, Tremblay J. Apoptosis in target organs of hypertension. Hypertension. 1995; 26: 642-48.

https://doi.org/10.1161/01.hyp.26.4.642

PMID:

36. Kruyer A, Soplop N, Strickland S, Norris EH. Chronic Hypertension Leads to Neurodegeneration in the TgSwDI Mouse Model of Alzheimer's Disease. Hypertension. 2015; 66:175-82. https://doi.org/10.1161/HYPERTENSIONAHA.115.0552 4 PMID:25941345 
37. Manolio TA, Olson J, Longstreth WT. Hypertension and cognitive function: pathophysiologic effects of hypertension on the brain. Curr Hypertens Rep. 2003; 5:255-61.

https://doi.org/10.1007/s11906-003-0029-6

PMID:12724059

38. van Assche T, Buys R, de Jaeger M, Coeckelberghs $E$, Cornelissen VA. One single bout of low-intensity isometric handgrip exercise reduces blood pressure in healthy pre- and hypertensive individuals. J Sports Med Phys Fitness. 2017; 57:469-75.

https://doi.org/10.23736/S0022-4707.16.06239-3

PMID:27029960

39. Shin MS, Jeong HY, An DI, Lee HY, Sung YH. Treadmill exercise facilitates synaptic plasticity on dopaminergic neurons and fibers in the mouse model with Parkinson's disease. Neurosci Lett. 2016; 621:28-33. https://doi.org/10.1016/j.neulet.2016.04.015 PMID:27080424

40. Sung YH, Kim SC, Hong HP, Park CY, Shin MS, Kim CJ, Seo JH, Kim DY, Kim DJ, Cho HJ. Treadmill exercise ameliorates dopaminergic neuronal loss through suppressing microglial activation in Parkinson's disease mice. Life Sci. 2012; 91:1309-16.

https://doi.org/10.1016/j.Ifs.2012.10.003

PMID:23069581

41. Krantic S, Mechawar N, Reix S, Quirion R. Apoptosisinducing factor: a matter of neuron life and death. Prog Neurobiol. 2007; 81:179-96.

https://doi.org/10.1016/i.pneurobio.2006.12.002 PMID:17267093

42. Ackery A, Robins S, Fehlings MG. Inhibition of Fasmediated apoptosis through administration of soluble
Fas receptor improves functional outcome and reduces posttraumatic axonal degeneration after acute spinal cord injury. J Neurotrauma. 2006; 23:604-16.

https://doi.org/10.1089/neu.2006.23.604

PMID:16689665

43. Upadhyay D, Panduri V, Ghio A, Kamp DW. Particulate matter induces alveolar epithelial cell DNA damage and apoptosis: role of free radicals and the mitochondria. Am J Respir Cell Mol Biol. 2003; 29: 180-87.

https://doi.org/10.1165/rcmb.2002-02690C PMID:12600817

44. Mazarakis ND, Edwards AD, Mehmet H. Apoptosis in neural development and disease. Arch Dis Child Fetal Neonatal Ed. 1997; 77:F165-70.

https://doi.org/10.1136/fn.77.3.f165

PMID: 9462183

45. Kim JH, Liu QF, Urnuhsaikhan E, Jeong HJ, Jeon MY, Jeon S. Moderate-Intensity Exercise Induces Neurogenesis and Improves Cognition in Old Mice by Upregulating Hippocampal Hippocalcin, Otub1, and Spectrin- $\alpha$. Mol Neurobiol. 2019; 56:3069-78. https://doi.org/10.1007/s12035-018-1239-x PMID:30091036

46. Griesbach GS, Hovda DA, Molteni R, Wu A, GomezPinilla F. Voluntary exercise following traumatic brain injury: brain-derived neurotrophic factor upregulation and recovery of function. Neuroscience. 2004; 125:129-39.

https://doi.org/10.1016/j.neuroscience.2004.01.030 PMID:15051152 